\title{
Surgical Management of Intraoperative Aortic Dissection in Type 3 Loeys-Dietz Syndrome with MYH11 Co-Mutation
}

\author{
Fumiya Yoneyama, MD, Hideyuki Kato, MD, PhD, Bryan J. Mathis, PhD, Yuji Hiramatsu, MD, PhD \\ Department of Cardiovascular Surgery, University of Tsukuba, Tsukuba, Japan
}

\section{ABSTRACT}

A 57-year-old female with Loeys-Dietz syndrome type 3 and MYH11 gene mutation underwent unexpected intraoperative ascending aortic dissection and subsequent rapid progression of the aortic arch dissecting aneurysm. Intra-surgical contingency plans with regard to aortic dissection and aneurysm should be considered for Loeys-Dietz syndrome, especially with comorbid mutations.

\section{INTRODUCTION}

Aortic dissection and aneurysmal formation due to aortic fragility are signature pathologies of Loeys-Dietz syndrome (LDS) but the type 3 variant is more known for mitral valve dysfunction than aortic issues [Krohg-Sørensen 2017]. However, comorbid genetic mutations involved in hereditary aortic aneurysm and dissection, such as MYH11, will exacerbate aortic fragility [Takeda 2019; Ikeda 2016]. Numerous reports have detailed the fragility of the LDS aorta but each incidence may possess unique factors that must be addressed with surgical planning that allows for rapid containment or replacement of the damaged vasculature. Here, we describe a case of Loeys-Dietz syndrome type 3 bearing a MYH11 mutation, in which ascending aortic cannulation caused an intraoperative ascending aortic dissection and rapid progression of the dilated aortic arch and descending aorta. Prompt and effective surgical intervention both repaired the aortic damage and prevented further life-threatening adverse events.

\section{CASE REPORT}

A 57-year-old female was referred to our hospital due to severe mitral regurgitation. Genetic testing at another institute revealed gene mutations in SMAD3 for LDS type 3 (LDS3) and MYH11 for familial thoracic aortic aneurysm and dissection (TAAD). Echocardiography showed left ventricular dilation, with a poor left ventricular ejection fraction (LVEF) of 37\%, while the mitral valve anterior and posterior

Received December 10, 2020; accepted fanuary 3, 2021.

Correspondence: Hideyuki Kato, $M D, P b D$, Department of Cardiovascular Surgery, University of Tsukuba, 1-1-1 Tennodai, Tsukuba 305-8575, Japan; +81-29-853-3210; fax: +81-29-853-3097 (e-mail: bkato@md.tsukuba.ac.jp). leaflets were billowing, causing severe regurgitation. A mitral valve reconstruction was scheduled.

After median sternotomy, we confirmed an intact ascending aorta by transesophageal echocardiography before cannulating it $(21 \mathrm{Fr})$ along with bicaval cannulations. Once cardiopulmonary bypass $(\mathrm{CPB})$ was initiated, an aortic dissection was

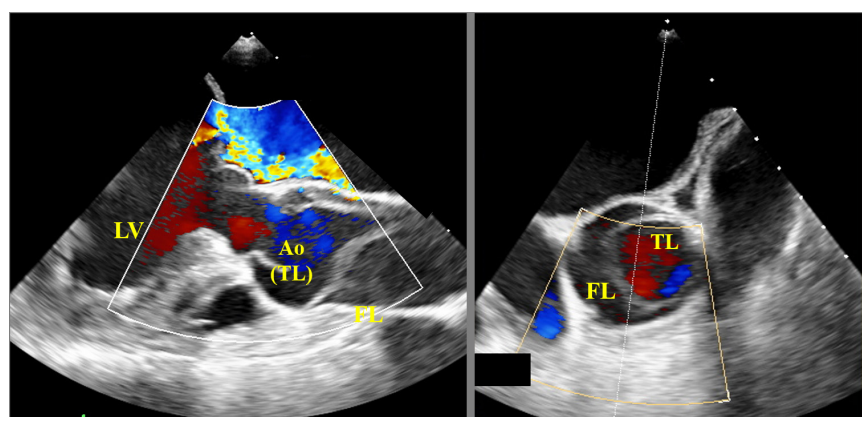

Figure 1. Transesophageal echocardiography showing ascending aortic dissection. Ao, aorta; TL, true lumen; FL, false lumen; LV, left ventricle

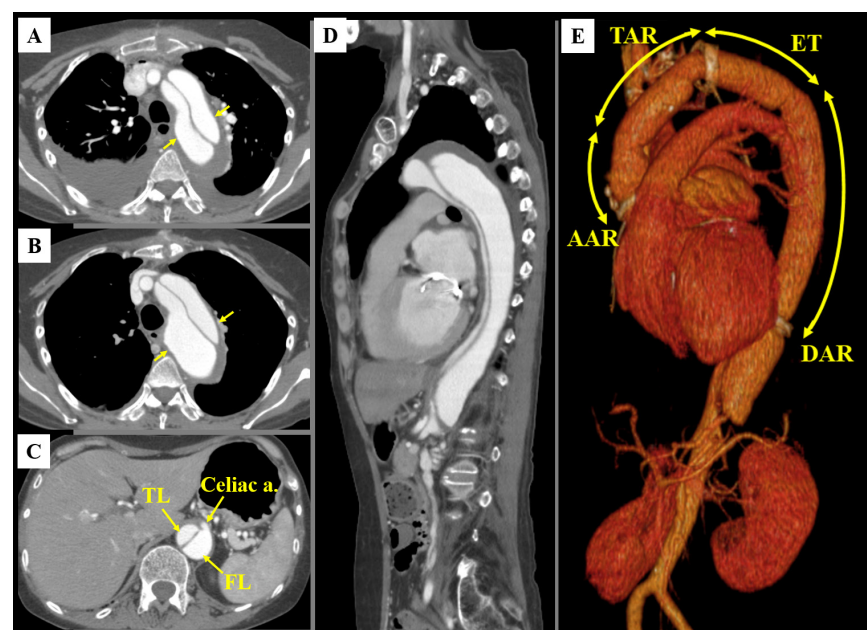

Figure 2. Postoperative computed tomography. A) The distal aortic arch enlarged at one month after surgery. B) The progressively enlarged aorta at two months after surgery. C) The celiac artery here is supplied from the false lumen of the dissected aorta. D) The aorta was dissected from the thoracic to the abdominal area. E) Three-dimensional imaging of the aorta after a surgical series. AAR, ascending aortic arch replacement; Celiac a., celiac artery; DAA, descending aorta replacement; ET, elephant trunk; FL, false lumen; TAA, total arch replacement; TL, true lumen 
observed to have spread from the ascending to the descending aorta (Figure 1) and, in response, the common femoral artery was immediately exposed and cannulated (16Fr) to switch sites. (Figure 1) After aortic incision under hypothermic circulatory arrest, dissected aortic wall fragments were observed at the cannulation site of the ascending aorta. Because the extent of the aortic dissection was unknown, we decided to perform an ascending aorta replacement (AAR) as a salvage plan for the unexpected, life-threatening event. AAR was performed with a $24 \mathrm{~mm}$ artificial graft while mitral valve replacement was conducted with a $29 \mathrm{~mm}$ tissue valve. Despite an uneventful postoperative course, vigilance against a dissecting aortic arch was maintained. Computed tomography (CT) demonstrated rapid dilation of the dissected distal aortic arch and descending aorta, $50 \mathrm{~mm}$ in diameter with a $10 \mathrm{~mm}$ increase over 4 weeks (Figure 2A, B, and D), while the celiac artery was being supplied from the false lumen (Figure 2C). This rapid progression indicated total arch replacement (TAR) and descending aorta replacement (DAR) within a short span to prevent aortic rupture but an LVEF of $35 \%$ mandated a stepwise correction. Thoracic endovascular aortic repair was evaluated as an option for treatment but was abandoned due to risk of ischemia in the celiac artery branching from the false lumen.

TAR was performed first with CPB initiation via right axillary arterial grafting and right atrial cannulation. Distal anastomosis was performed utilizing an elephant trunk technique with a $22 \mathrm{~mm}$ artificial graft. As the celiac artery was supplied from the false lumen, a fenestration was created by removing the intra-aortic flap of the distal aortic arch. The elephant trunk thus easily enabled clamp access for the next DAR.

Two days later, DAR was performed and this time CPB was established via femoral arterial and venous cannulation. With cardiac beating on $\mathrm{CPB}$, the previously inserted elephant trunk was identified and clamped via left thoracotomy. Descending aortic clamping was avoided to prevent wall injury and, instead, a 16Fr Foley catheter was inserted into the true lumen of the descending aorta for distal anastomosis. DAR was performed with a $24 \mathrm{~mm}$ artificial graft. Patient recovery was uneventful and postoperative $\mathrm{CT}$ revealed a successful reconstruction (Figure 2E).

\section{DISCUSSION}

Intraoperative aortic dissection is an extremely rare (0.04$0.35 \%$ ) but lethal surgical complication [Narayan 2015] and connective tissue disorders, such as Marfan syndrome and LDS, are known to increase risk due to associated aortic wall fragility [Narayan 2015; Kumar 2009]. Although the genetic etiology is substantially heterogenous and approximately $80 \%$ of all familial non-syndromic TAAD cannot be explained by pathogenic variants, the ClinGen Aortopathy Expert Panel classified 11 causative genes (FBN1, TGFBR1, TGFBR2, SMAD3, TGFB2, COL3A1, ACTA2, MYH11, MYLK, LOX, and PRKG1) for hereditary TAAD as category
A [Takeda 2019]. Since the smooth muscle-specific MYH protein imparts contractile function, MYH11 heterogenous mutations lead to an early and severe decrease in the elasticity of the aortic wall. Histologic features of these variant genotypes are elastic fiber fragmentation and loss, decreased smooth muscle cell number, cystic medial degeneration, and fibromuscular dysplasia but the most pathogenic variants of MYH11 have been found in patients with TAAD exhibiting patent ductus arteriosus [Ikeda 2016]. To the best of our knowledge, there are no other reports of an LDS case with a MYH11 co-mutation. Although extremely rare, significant aortic wall fragility in these patients is an expected phenotype and extra caution should have been taken in this case.

In our case, LDS3 and the comorbid MYH11 mutation for TAAD we encountered were a red flag for complications, requiring rapid intra- and postoperative strategies to compensate for unpredictable vascular damage and facilitate subsequent operations. Ideal approaches to arterial cannulation in LDS are scarcely reported; however, aortic dissection risk, especially in those with co-morbid category A mutations, should be considered even if LDS3 cases are traditionally mitral and not aortic-focused. In addition, direct manipulations in these cases, such as aortic direct cannulation or cross clamping, might be contraindicated due to aortic wall fragility. As preservation of aortic wall integrity is of paramount importance, adaptive repair strategies for intraoperative dissections and vigilance against postoperative complications in LDS patients suffering from connective tissue diseases must be prioritized in surgical planning.

\section{CONCLUSION}

Intra- and postoperative strategies regarding unpredictable aortic dissection and aneurysm should be considered for Loeys-Dietz syndrome, especially with comorbid genetic mutations. Direct aortic manipulations should be avoided due to aortic wall fragility.

\section{REFERENCES}

Krohg-Sørensen K, Lingaas PS, Lundblad R, et al. 2017. Cardiovascular surgery in Loeys-Dietz syndrome types 1-4. Eur J Cardiothorac Surg. 52:1125-31.

Kumar K, Menkis AH, Jassal DS, Arora RC. 2009. Iatrogenic acute aortic dissection in a patient with Marfan syndrome: unusual site of intimal tear. Interact Cardiovasc Thorac Surg. 8:362-3.

Ikeda Y. 2016. Aortic Aneurysm: Etiopathogenesis and Clinicopathologic Correlations. Ann Vasc Dis. 9:73-9.

Narayan P, Angelini GD, Bryan AJ. 2015. Iatrogenic intraoperative type A aortic dissection following cardiac surgery. Asian Cardiovasc Thorac Ann. 23:31-5.

Takeda N, Komuro I. 2019. Genetic basis of hereditary thoracic aortic aneurysms and dissections. J Cardiol. 74:136-43. 